\title{
Introducing the Journal of Population Ageing
}

\author{
Sarah Harper • George Leeson
}

Published online: 27 May 2009

(C) The Author(s) 2009. This article is published with open access at Springerlink.com

The second half of the 20th century saw the more developed countries of the world experience population ageing to a degree hitherto unseen in demographic history. The first half of the $21^{\text {st }}$ Century is predicted to see the same transition within the less developed and transitional countries. Globally, by 2050 there will be some 2 billion adults aged over 60 , and the total number of older people will outnumber the young. This is historically unprecedented. It makes the $20^{\text {th }}$ century the last century of youth, the $21^{\text {st }}$ century the first of population maturity.

Most western style countries have aged continuously over the past century, the measure of ageing being an increase in the percentage of those over 60 years, and a decrease in those under 15 years. Europe reached maturity at the turn of the millennium, with more older people than younger. By 2030 half the population of Western Europe will be over $50,25 \%$ over 65 , and $15 \%$ over 75 . By 2030 one quarter of the population of the developed world will be over 65 , and by the middle of the century this will have risen to one third. Yet while most interest has focused on the ageing of Europe, it is the Asian/Pacific region that is ageing most rapidly. By 2030 one quarter of the population of Asia will be over 60, and by 2040 Asia will be demographically mature, with more older than younger people. If we move from structural ageing to consider absolute numbers of older people, the dominance of the less developed regions, and in particular Asia, becomes even more apparent. Already two thirds of the world's older population live in less developed regions with the absolute numbers of older people in these regions doubling to reach some 900 million within 25 years. By 2050 two-thirds of the world's elders will live in Asia alone.

The numbers of those aged 80 and above will show an even greater increase, rising from 69 million to near 400 million by 2050 . Thus by the middle of the 21 st Century there will be almost as many over $80 \mathrm{~s}$ as there were over $65 \mathrm{~s}$ at the beginning. This is the fastest growing age group in the world with an annual growth rate of $3.8 \%$. Low fertility around the time of the First World War and declining

S. Harper $(\triangle) \cdot$ G. Leeson

Oxford Institute of Ageing, University of Oxford, Parks Road, Oxford OX1, UK

e-mail: sarah.harper@ageing.ox.ac.uk 
mortality rates among this cohort partially explain this-people reaching 80 in the mid-1990s were part of a relatively small birth cohort. Fertility increased again within a few years and at the turn of the century a much larger birth cohort was reaching 80 . However, the projected annual growth rate of this age group remains above $3 \%$ until at least 2020, in comparison with $2 \%$ for the over 65 group as a whole. By the year 2050, 20\% of persons aged 60 and over are predicted to be in this group. Currently $40 \%$ of the over 80 s live in Asia, some $16 \%$ in China alone, partly a reflection of China's very large proportion of the total world population; $30 \%$ are in Europe and $13 \%$ in the US. Japan is predicted to have $40 \%$ of its older population 80 or over by 2030 . The number of centenarians will increase equally dramatically, with a predicted 25 million globally by the mid century.

Similarly, it is the less developed and transitional countries which will face extreme rapidity of ageing. While it took Europe (EU 15) some 120 years to go from a young to mature population, such a shift in the proportion of young and old will have occurred in Asia in less than 25 years. France, for example, took 115 years to move from $7 \%$ to $14 \%$ of its population over 65 , the UK achieved this in only 45 years, and Japan in 26 . While the predicted increase in the percentage of people over 60 by 2025 for the EU 15 is around 33\%, it is a staggering 400\% for Indonesia, $350 \%$ for Thailand, Kenya and Mexico, 280\% for Zimbabwe and up to $250 \%$ for India, China and Brazil. It is this rapidity of demographic ageing which will be key in the policy changes needed within less developed and transitional regions.

The demographic situation in most developed, and a growing number of transitional countries is thus defined principally by the combination of three dominant trends: fertility rates at or below replacement levels, unprecedented and continuing declines in late-life mortality, and increasing levels of migration. This is resulting in societies which are increasingly characterised by a decline in the proportion of younger people (through falling fertility), an increase in the proportion and number of older people (through both falling fertility and mortality), and a more ethnically diverse composition (through increased migration).

One of the key drivers of population ageing is the fall in Total Fertility Rates (TFR). Alongside the well recognised low fertility of Europe, we see a similar pattern emerging in Asia. Singapore and Korea have now fallen to below 1.2, while Hong Kong at below 1 now has the lowest TRF in the world. In terms of mortality fall, the most striking feature of life expectancies at birth is not only that everyone born in the developed world, with the exception of the former Eastern European countries, can now expect to live for more than 75 years, but also the high life expectancies at birth now expected in much of Asia and Latin America. Indeed with the exception of Africa, many countries in the less developed world now have life expectancies at birth of 70 years or older, and life expectancies at age 60 of a further 15 to 20 years, which are comparable with European ones. The second demographic transition of developed countries is thus being mirrored in other parts of the world, particularly Asia, as fertility falls from the replacement levels of classic demographic transition theory, and globally mediated by the third demographic transition driven by international migration.

It is currently uncertain how low fertility will fall in Europe and some of the more advanced Asian countries. A combination of further declining family size ideals, continued postponement of childbearing and bio-medical factors affecting both men and women may well lead to fertility levels so far below replacement level as to have 
dramatic consequences for the social and economic structures of society. The recently proposed "Low Fertility Trap Hypothesis" assumes a bifurcation among industrialized countries under which the lowest fertility countries would see further fertility declines while another set of countries would experience stable fertility only slightly below replacement level. Alongside this lie new perspectives on mortality forecasting, which acknowledge that there is much greater scope for reductions in mortality at higher ages than previously acknowledged. Important as these national and/or regional concerns with population ageing and low fertility are, we have still to ask how they relate to continuing concern about unsustainable global population growth. If this century will indeed witness the end of world population growth a key question arises as to whether it is right to let the concerns of neo-Malthusians fall off the political agenda of the wealthier nations of the world, or whether this should remain one of the fundamental challenges for our collective future.

It is also unclear as to how these trends will develop over the course of this century. In the developed world, for example, there are relatively large birth cohorts now in midlife, that are longer-lived and have lower fertility than their parents. These three factors mean that their entry into old age will generate what is sometimes described as an 'age wave' or 'demographic shock' that will subsequently subside as smaller cohorts take their place. Old age dependency ratios will increase sharply as the consequences of rapid and large declines in fertility work themselves through the population. However, the continued increases in longevity, including potential radical increases due to new generation technological advances, accompanied by persistent falls in fertility, mean that the population structure of both the developed and developing regions may well alter even more dramatically over the course of this century.

Such global ageing, however, is not occurring in isolation, it is emerging in the context of globalisation itself, a world increasingly dominated by the flow of human and economic capital across national boundaries. Indeed, a key stimulus to such capital flows is the emerging demographic imbalances arising from the differential movement of regions into maturity. Thus while an understanding of the dynamics of globalisation is essential to address the challenges and opportunities of ageing societies, so it is also necessary to understand the dynamics of global ageing as a component of globalisation.

It was with this demographic context in mind that the Oxford Institute of Ageing was established at the University of Oxford in 2001, a multi-disciplinary research institute drawing together researchers from across the social sciences-economics, statistics, demography, anthropology, sociology, psychology, law and history-to work with colleagues from biology, neuroscience, public health, medicine, engineering and technology to examine global societal ageing. The Journal of Population Ageing (JPA) was developed out of these interactions, and its focus thus reflects the emphasis at Oxford on a multi-disciplinary approach to understanding the processes and interconnections behind the drivers and outcomes of demographic ageing. JPA will address the broad questions of ageing societies internationally, thereby stimulating discussion of North American, European, and Transitional and Developing World issues.

A key question, for example, is whether we should expect demographic convergence to accompany socio-economic convergence and the role that migration has to play in this process. As restrictions on the movement of human and financial capital around the world are eased, demographic change becomes a potent force for change in the global economy. Exactly how these changes will play out remains poorly understood. Large 
shifts in national age distributions are likely to affect national saving patterns, capital requirements and international capital flows, particularly between the more and less developed worlds. The demand for health and social care workers in more developed countries is already increasing, and is set to increase further at the same time as the supply of younger workers will tighten. The implications for the host and source countries' welfare systems, and for the family and social support structures in the source countries, are considerable. The future promises many similar scenarios across many different sectors of the national economy, with skilled labour being pulled out of the country as well as pulled in. Broader questions include to what extent can and should immigration mitigate certain negative effects of demographic ageing; what policies should be developed for better integrating these migrants, in particular young people; how could the legislative and financial frameworks and incentives combat discrimination and promote integration of immigrants?

Another key area of debate concerns the impact of population ageing on the intergenerational transfer of resources. Most countries in the world have developed public institutions for transferring resources and support between working generations to dependent younger and older generations. Population ageing is bringing about such large changes in the relative size of these generational groupings that policy-makers have to reconsider the operation of the institutions that channel public resources and support between generations. In addition, declining fertility affects the collective capacity of society to provide these goods and assist with the problems that face the ageing individual.

We also need to consider the adjustments which will have to be made for a lowmortality and low-fertility future. While policy makers recognise that they have to help their societies adjust to a low-mortality and low-fertility future, they are unclear as to how large these adjustments will have to be. The adjustments required in order to finance the additional consumption of longer-lived populations under conditions of declining fertility clearly pose major allocation and distributional challenges. For example, as individuals we may be required to reconsider the way in which we allocate consumption and resources between different stages of the life course; as societies, we have to decide how to allocate the burden of adjusting to demographic change across both different parts of the life course and between different generations. However, current assumptions on longevity may turn out to be too conservative due, for example, to the speed of technological advance in biomedicine, or indeed, too optimistic due, for example, to the increasing prevalence of obesity. The extreme scenarios we now have to consider include the possibility that biomedicine will enable young children today to remain active and healthy as centenarians as well as the possibility that their life expectancy will be less than that of their parents. What will be the role of the new genetics and bio-technology in extending lives?

Of equal importance is the debate over how governments and employers will enable individuals to maintain their health and productive capacity. The labour market will face both increasing skills shortages as well as a large proportion of older workers, and will need to adapt to train and retain older workers. New cohorts will expect and demand increasingly flexible working patterns. Home is likely to develop as a place of work, education and health care. We need to consider how the organisation of work best be adapted to a new distribution between the generations, with fewer young people and great numbers of older workers, to take into account the specific needs of different age groups; how parents' integration in working life can be facilitated and how they can 
achieve a balance between flexibility and security to bring up their children, to train and update their skills to meet the demands of the labour market. Societies will need to decide what is an appropriate balance between investing in early education, and in adult and lifelong training schemes.

The questions are numerous. The family as a supportive environment will change, though how is unclear. We will move increasingly into second, third and even fourth partnerships with extended families of a complicated and demanding nature. As more generations will survive next to each other than ever before, people will increasingly pass income, care and support down as well as up through the generations. What will be the new forms of intergenerational solidarity as intergenerational transfers and justice move to the fore of policy concern and will influence the new ethics of our societies, how will this affect the inter-generational contract?

Communities will change both spatially and socially. How will technology enhance our future lives? How will our living and working environments respond to the needs of a more mature population? How will individual life courses change, both professionally and personally, as we recognise our personal longevity? Individuals will have to rethink their own personal life courses and when and how they wish to mix education and work. How will societal structure and organisation change to keep up with the new demographic reality? How will social and economic behaviour adapt? Consumption will vary between ages groups and generations and will not be the same as that of previous generations. How will future generations adjust their savings and consumption behaviour across the life course? And how will governments and private sector providers maintain the capacity to provide late life financial security? How will infrastructure and services, such as housing and transport, adapt to a large percentage of older adults' needs and capacities?

How will transitional and less developed societies provide the financial and social care infrastructures appropriate for these economies, welfare systems and cultures and in particular how will their health care systems adapt to a reduction in acute diseases and infant and child related medicine, and to non-communicable disease and long term care.

There are clearly huge challenges ahead, but also real opportunities-age integrated flexible workforces, intergenerational extension, age equality, and politically stable, age integrated societies are all potential benefits of demographically mature societies. The growth of societies across the globe in which the majority of all individuals born grow up to live long, healthy, active lives has the possibility to be one of the major successes of the $21^{\text {st }}$ Century. Drawing on its Oxford heritage of a multi-disciplinary approach to understanding the processes and interconnections behind the drivers and outcomes of demographic ageing, it is hoped that the Journal of Population Ageing (JPA) will thus provide a forum for international, cross-disciplinary debate on population ageing which will lead to a greater understanding of the challenges and opportunities of this new mature world.

Oxford

March 2009

Open Access This article is distributed under the terms of the Creative Commons Attribution Noncommercial License which permits any noncommercial use, distribution, and reproduction in any medium, provided the original author(s) and source are credited. 